Please do not remove this page

RMIT

UNIVERSITY

\title{
Photonic reconfigurable microwave filter with negative coefficients
}

Mansoori, Sana; Mitchell, Arnan; Ghorbani, Kamran

https://researchrepository.rmit.edu.au/esploro/outputs/9921857632301341/filesAndLinks?institution=61RMIT_INST\&index=null

Mansoori, S., Mitchell, A., \& Ghorbani, K. (2004). Photonic reconfigurable microwave filter with negative coefficients. Electronics Letters, 40(9), 541-543. https://doi.org/10.1049/el:20040364

Published Version: https://doi.org/10.1049/el:20040364

Repository homepage: https://researchrepository.rmit.edu.au

(c) IEEE 2004. Personal use of this material is permitted. However, permission to reprint/republish this material for advertising or promotional purposes or for creating new collective works for resale or redistribution to servers or lists, or to reuse any copyrighted component of this work in other works must be obtained from the IEEE.

Downloaded On 2023/04/27 00:41:58 +1000 


\section{Photonic reconfigurable microwave filter} with negative coefficients

\section{S. Mansoori, A. Mitchell and K. Ghorbani}

A photonic implementation of a reconfigurable microwave transversal filter is proposed. The implementation allows the filter taps to be both positive and negative. This allows for greater flexibility in the design of the filter so that bandpass and lowpass filters with flatter passbands could be realised. A four-tap version of the filter is demonstrated.

Introduction: Optically realised microwave filters offer advantages such as large operating bandwidth and immunity to microwave interference. Several researchers have investigated microwave photonic filters to enable tunable and reconfigurable systems. Capmany et al. [1] proposed a filter system that employs an array of tunable lasers. The filter can be both tuned and reconfigured by changing the wavelength separation and optical power, respectively. Vidal et al. [2] proposed a modification that replaced the array of tunable lasers with a single multiwavelength source. Discrete tuning is achieved using a switched dispersion matrix. This is a more economical solution but the filter taps are limited to positive coefficients. This restricts the filters to only lowpass and also restricts the flexibility in the design of the lowpass filters.

Several techniques have been proposed to implement negative coefficients. Two-tap bandpass filters using all optical techniques have been demonstrated in [3-5]. Electro-optic techniques have been reported in [6] and [7]. However, these require separate arrays of tunable lasers to implement each of the set of positive and negative coefficients.

In this Letter, we propose a modification to the filter proposed in [2]. The implementation allows for both positive and negative taps using a single wavelength selective element. A four-tap version of the filter is demonstrated.

Principle of operation: Fig. 1 shows a block diagram of the proposed microwave transversal filter. The optical carrier is provided by a single multiwavelength source. The optical power is divided into two arms using a 2/3:1/3 directional coupler. Each arm contains an electrooptic modulator. One modulator is biased at positive quadrature (+ve arm) and the other is biased at negative quadrature (-ve arm). The optical signal in the + ve arm travels through a reconfigurable optical filter (ROF) to shape the signal. The signals from the two arms are then combined and launched into a dispersive medium to obtain the wavelength dependent delays. The detected RF signal at the output of the dispersive medium is a filtered version of the input RF signal.

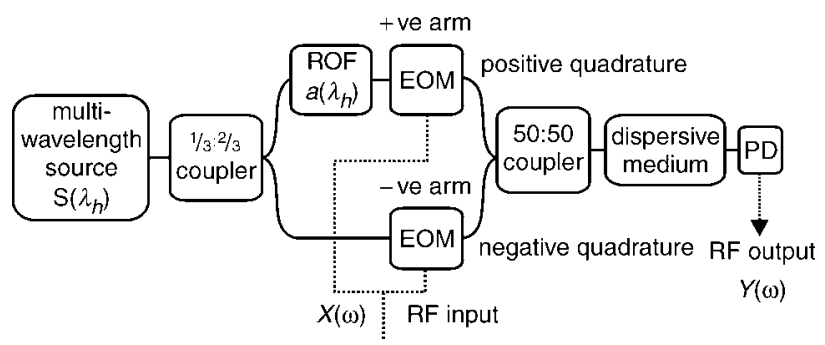

Fig. 1 Block diagram of proposed transversal filter

From Fig. 1, the transfer function of the transversal filter is summarised in the following expression:

$$
H(\omega)=\sum_{n=0}^{N-1} S\left(\lambda_{n}\right)\left(\frac{2}{3} a\left(\lambda_{n}\right)-\frac{1}{3}\right) e^{-j \omega \tau_{n}}
$$

where $N$ is the number of carrier wavelengths, $\lambda_{n}$ is the $n$ the wavelength of the optical carrier, $S\left(\lambda_{n}\right)$ is the optical power at the source, $a\left(\lambda_{n}\right)$ is the transfer function of the ROF, $\tau_{n}$ is the time delay experienced by $\lambda_{n}$ through the dispersive medium, and $\omega$ is the RF frequency.

With no ROF attenuation of the power (i.e. $a\left(\lambda_{n}\right)=1$ for all $n$ ), all the coefficients (taps) are positive with amplitude of $1 / 3$. When the entire positive arm signal is attenuated (i.e. $a\left(\lambda_{n}\right)=0$ for all $n$ ), all the taps are negative. By setting the $a\left(\lambda_{n}\right)$ to values between 0 and 1 , the transfer function of the filter can be reconfigured to obtain any combination of positive and negative coefficients with amplitudes between $-1 / 3$ and $1 / 3$.

Experimental setup: To verify the proposed filter concept, a four-tap version of the filter was realised using the setup shown in Fig. 2. A WDM source and a dynamic channel equaliser (DCE) would be the preferable components for the multiwavelength source and the ROF, respectively, because they would have matching wavelength separations. However, the optical filter available was a dynamic gain equaliser (DGE) module. A DGE works as a wavelength dependent attenuator but has a broader channel separation than a DCE. Four tunable lasers set at equally spaced wavelengths of 1535, 1540, 1545 and $1550 \mathrm{~nm}$ were combined and used to simulate a WDM source with wavelength separation compatible with the DGE. The particular DGE used in the implementation has 26 channels with channel separation of $2 \mathrm{~nm}$ and a $3 \mathrm{~dB}$ bandwidth of $2.5 \mathrm{~nm}$ per channel. A cascade of three 50:50 couplers and a variable optical attenuator (VOA) in the negative arm were used to replace the $2 / 3: 1 / 3$ directional coupler. The VOA was set such that the optical power in the negative arm was approximately half the power in the positive arm. To avoid coherent interference when combining the signals from both arms, a polarisation beam splitter was used to combine the signals as described in [8]. A true time delay phase shifter (TTD-PS) was used in the negative arm to adjust the path-lengths.

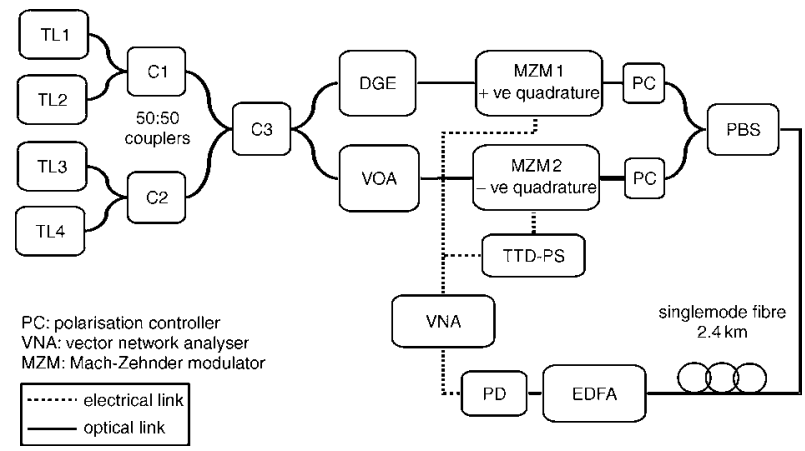

Fig. 2 Experiment setup

To use the system to realise a lowpass filter with a flat passband, the impulse response should approximate a sinc function. To approximate a sinc function using four coefficients, the two centre taps at 1540 and $1545 \mathrm{~nm}$ were set to equal positive values (no attenuation from the ROF at these wavelengths). Using the DGE, the optical power in the positive arms at wavelengths 1535 and $1550 \mathrm{~nm}$ were attenuated to obtain negative coefficients at these wavelengths, as shown in Fig. 3. By controlling the amount of attenuation at these wavelengths different ratios of positive to negative taps were obtained corresponding to different apodisations of the impulse response.

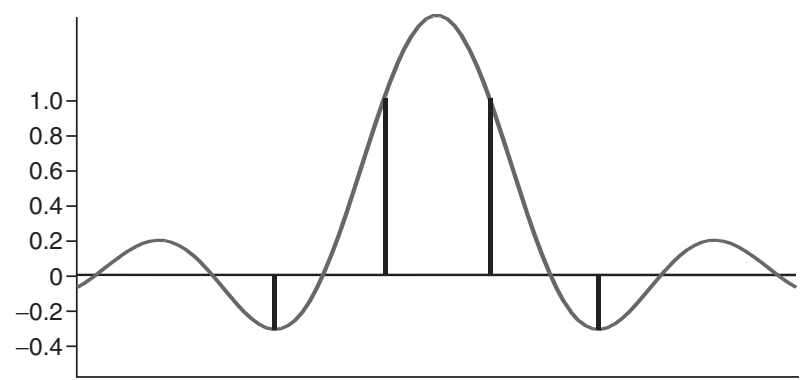

Fig. 3 Four taps approximating a sinc impulse response

Results and discussions: Fig. 4 shows the experimental results obtained for positive to negative tap ratios of $(a)$ 1:0.13, (b) 1:0.18 and $(c) 1: 0.22$. The experimental results are plotted in the dotted lines and theoretical predictions are plotted in the solid lines for comparison. Good agreement is evident. The amplitude responses in the three traces show flat passbands that can only be realised using both positive and negative coefficients. The passband ripple was $(a)$ $0.05 \mathrm{~dB},(b) 0.5 \mathrm{~dB}$ and $(c) 1 \mathrm{~dB}$. The roll-off becomes steeper with increased ripple, as expected. The results demonstrate the feasibility 
of obtaining negative taps using this structure. Adjustments of the negative coefficients demonstrate that different apodisations of the impulse response can be achieved. Thus the passband ripple and filter roll-off can be reconfigured.
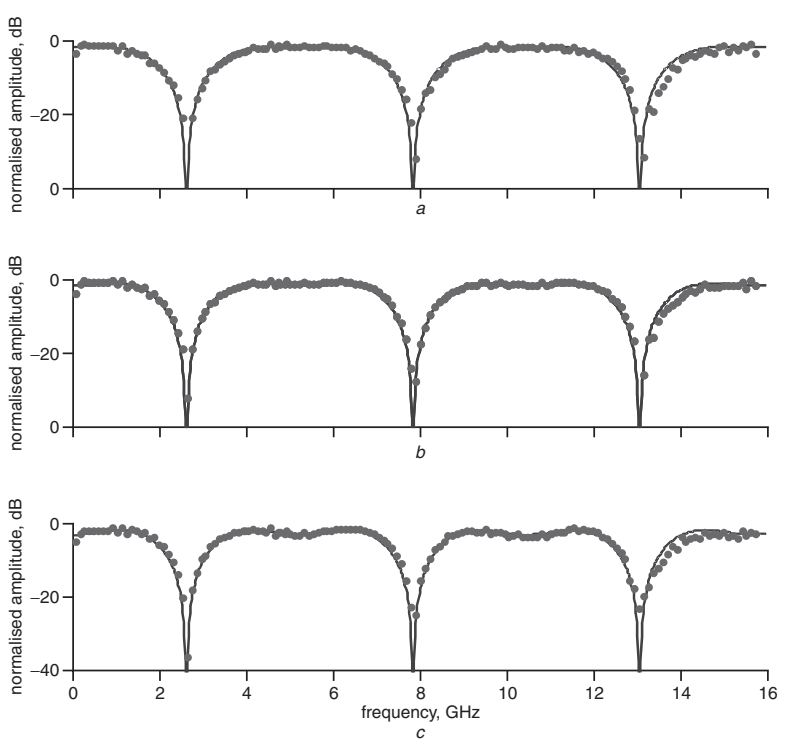

Fig. 4 Measured and predicted amplitude response of photonic transversal filter

$a$ Taps $=\left[\begin{array}{lllll}-0.13 & 1.0 & 1.0 & -0.13\end{array}\right] \quad b$ Taps $=\left[\begin{array}{lllll}-0.18 & 1.0 & 1.0 & -0.18\end{array}\right]$

$c$ Taps $=\left[\begin{array}{lll}-0.22 & 1.0 & 1.0\end{array}-0.22\right]$

c. Teasured

The advantage of the proposed filter is that a single source and ROF is used for both positive and negative arms and therefore the same taps (wavelengths) can be set to either positive or negative values. The filter can be reconfigured by simply adjusting the parameters of the ROF, which can be achieved digitally. However, the system operation is based on the cancellation of intensity modulation from both arms, which results in a reduction in the optical link gain.

Discrete tuning of the filter could be accomplished through the use of a switched dispersion matrix as described in [2]. Continuous tuning could be carried out using a tunable multiwavelength source in which case a more flexible ROF would be required.
Conclusions: A reconfigurable photonically realised RF transversal filter is proposed, which allows for both positive and negative coefficients using a single wavelength selective element. A four-tap version of the filter has been demonstrated showing the feasibility of realising lowpass filters with flatter response and sharper roll-off factors than is possible with only positive coefficients. The filter has the advantage of using a single power multiwavelength optical source and reconfigurable optical filter for both positive and negative taps but this comes at the cost of reduced link gain. Investigation of the proposed filter using a WDM source and a DCE will be conducted in the future.

(C) IEE 2004

Electronics Letters online no: 20040364

19 February 2004 doi: 10.1049/el:20040364

S. Mansoori, A. Mitchell and K. Ghorbani (Australian Photonics CRC, RMIT University, GPO Box 2476V, Melbourne, VIC 3001, Australia)

\section{References}

1 Capmany, J., Pastor, D., and Ortega, B.: 'New and flexible fiber-optic delay-line filters using chirped bragg gratings and laser arrays', IEEE Trans. Microw. Theory Tech., 1999, 47, pp. 1321-1326

2 Vidal, B., Polo, V., Corral, J.L., and Marti, J.: 'Photonic microwave filter with tuning and reconfiguration capabilities using optical switches and dispersive media', Electron. Lett., 2003, 39, pp. 547-548

3 Coppinger, F., et al.: 'All-optical incoherent negative taps for photonic signal processing', Electron. Lett., 1997, 33, pp. 973-975

4 Wang, X., and Chan, K.T.: 'Tunable all-optical incoherent bipolar delayline filter using injection-locked Fabry-Perot laser and fibre Bragg gratings', Electron. Lett., 2000, 36, pp. 2001-2003

$5 \mathrm{Li}, \mathrm{S}$., et al.: 'A novel tunable all-optical incoherent negative tap fiberoptic transversal filter based on a DFB laser diode and fiber Bragg gratings', IEEE Photonics Technol. Lett., 2000, 12, pp. 1207-1209

6 Sales, S., et al.: 'Experimental demonsttraion of fibre-optic delay line filters with negative coefficients', Electron. Lett., 1995, 31, pp. 10951096

7 Capmany, J., et al.: 'Using phase inversion in an electro-optic modulator to implement RF incoherent photonic filters with negative coefficients'. Int. Top. Mtg on Microwave Photonics, Budapest, Hungary, September 2003

8 Bui, L., Mitchell, A., and Chio, T.: 'Wideband vector sum phase shifter with minimal coherent interfernce'. COIN 2003 Conf. incorporating ACOFT, Melbourne, Australia, July 2003 